\title{
Amebiasis Masquerading Colonic Lymphoma in an Intestinal Intussusception Patient
}

\author{
Abdurrahman Sahina, g, Gamze Geyik ${ }^{\mathrm{b}}$, Turan Calhan ${ }^{\mathrm{c}}$, Resul Kahraman ${ }^{\mathrm{d}}$, Aysenur Ihvan ${ }^{\mathrm{e}}$, \\ Kamil Ozdilif, Haci Mehmet Sokmen ${ }^{\mathrm{f}}$
}

\begin{abstract}
Intussusception which presents with palpable mass, abdominal pain and bloody diarrhea is a rare condition in adulthood. Most of adult intussusception cases are secondary to benign or malign lesions. Entamoeba histolytica is a common pathogen leading to chronic bloody diarrhea in developing countries. We report a 22 -year-old patient presenting with abdominal pain and chronic bloody mucoid diarrhea, which intestinal amebiasis delayed diagnosis of intussusception secondary to colonic diffuse large B-cell lymphoma.
\end{abstract}

Keywords: Amebiasis; Colonic lymphoma; Intussusception

\section{Introduction}

Intussusception is one of the most common causes of acute abdomen in childhood. However, adult invagination is relatively rare and constitutes $5 \%$ of all intussusception cases [1]. Intussusception presents as classic clinical triad: palpable mass, abdominal pain and bloody mucoid stools. Although most of childhood intussusceptions are idiopathic, $90 \%$ of adult cases are secondary to benign or malign lesions $[2,3]$.

Manuscript accepted for publication July 7, 2014

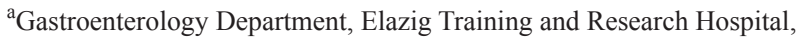
Elazig, Turkey

${ }^{\mathrm{b}}$ Department of Internal Medicine, Umraniye Training and Research Hospital, Istanbul, Turkey

${ }^{\mathrm{c}}$ Gastroenterology Department, Turkiye Gazetesi Hospital, Istanbul,

Turkey

${ }^{\mathrm{d}}$ Department of Gastroenterology, Batman State Hospital, Batman,

Turkey

e Department of Pathology, Umraniye Training and Research Hospital, Istanbul, Turkey

${ }^{\mathrm{f}}$ Department of Gastroenterology, Umraniye Training and Research

Hospital, Istanbul, Turkey

${ }^{g}$ Corresponding Author: Abdurrahman Sahin, Gastroenterology Department, Elazig Training and Research Hospital, Elazig, Turkey. Email: arahmansmd@yahoo.com

doi: http://dx.doi.org/10.14740/jmc1854w
Adult intussusception presents with nonspecific abdominal symptoms. It is important to diagnose and to differentiate from other inflammatory or mechanical obstructive disorders earlier.

Entamoeba histolytica (E. hisyolytica) infection becomes manifest as asymptomatic colonization, diarrhea, acute dysentery, fulminant colitis with perforation, toxic megacolon and ameboma formation in intestine. Intestinal amebiasis confused with many other benign or malignant diseases. We report here a patient with abdominal pain and bloody diarrhea which amebiasis masqueraded colonic diffuse large B-cell lymphoma presenting with ileocolonic invagination.

\section{Case Report}

A 22-year-old male presented with 6-week history of right lower side abdominal pain which had worsened over time and intermittent diarrhea. Patient had empirically been treated with PO ciprofloxacin. Recently, loss of appetite and recurrent vomiting were also added to his complaints and diarrhea had become bloody and mucoid. He denied any history of fever, noticeable weight loss or night sweats. His medical history had not any distinctive feature. On examination, the abdomen was soft, tender to palpation over the right lower quadrant with no rebound or guarding. There was no obvious

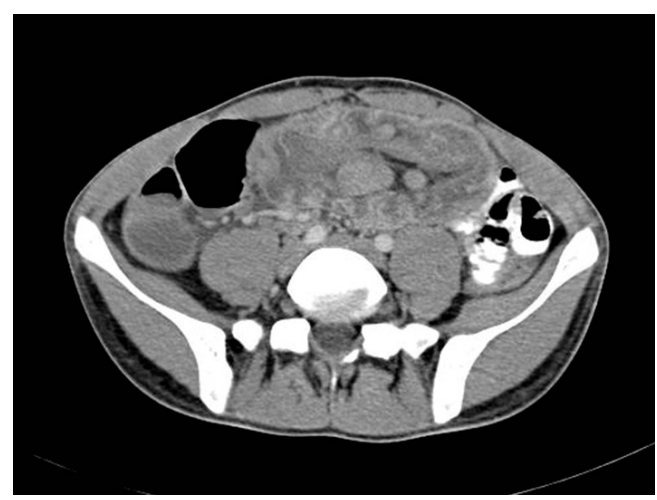

Figure 1. Computed tomography image of intussusception at midline with multiple lymphadenopathies. 


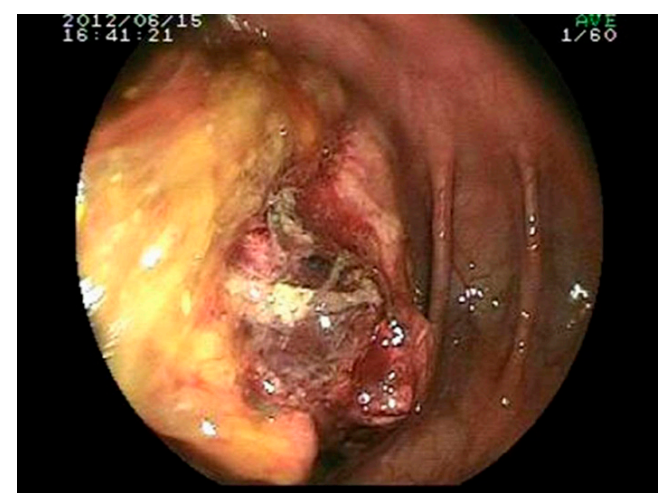

Figure 2. Mass lesion at the neighborhood of cecum on colonoscopic examination. mass lesion. Laboratory data revealed normal hemoglobin $(14 \mathrm{~g} / \mathrm{dL})$, white cell count $(9,810,000 / \mu \mathrm{L})$ and platelet count $(351,000,000 / \mu \mathrm{L})$. Erythrocyte sedimentation rate was 10 $\mathrm{mm} / \mathrm{h}$ and C-reactive protein was $0.54 \mathrm{mg} / \mathrm{dL}$. Urea, electrolytes, amylase and liver function tests were also normal, but lactate dehydrogenase was slightly raised (274 U/L (125 220)). Fecal leukocyte and erythrocyte was detected in stool examination. Stool antigen for E. histolytica was also positive. Intravenous fluids and metronidazole were started immediately and bloody diarrhea disappeared after treatment. However, colicky abdominal pain persisted and abdominal computed tomography with oral and intravenous contrast was performed. A 13-cm lesion with invaginated intestinal loops at the pelvic midline was detected (Fig. 1). There were
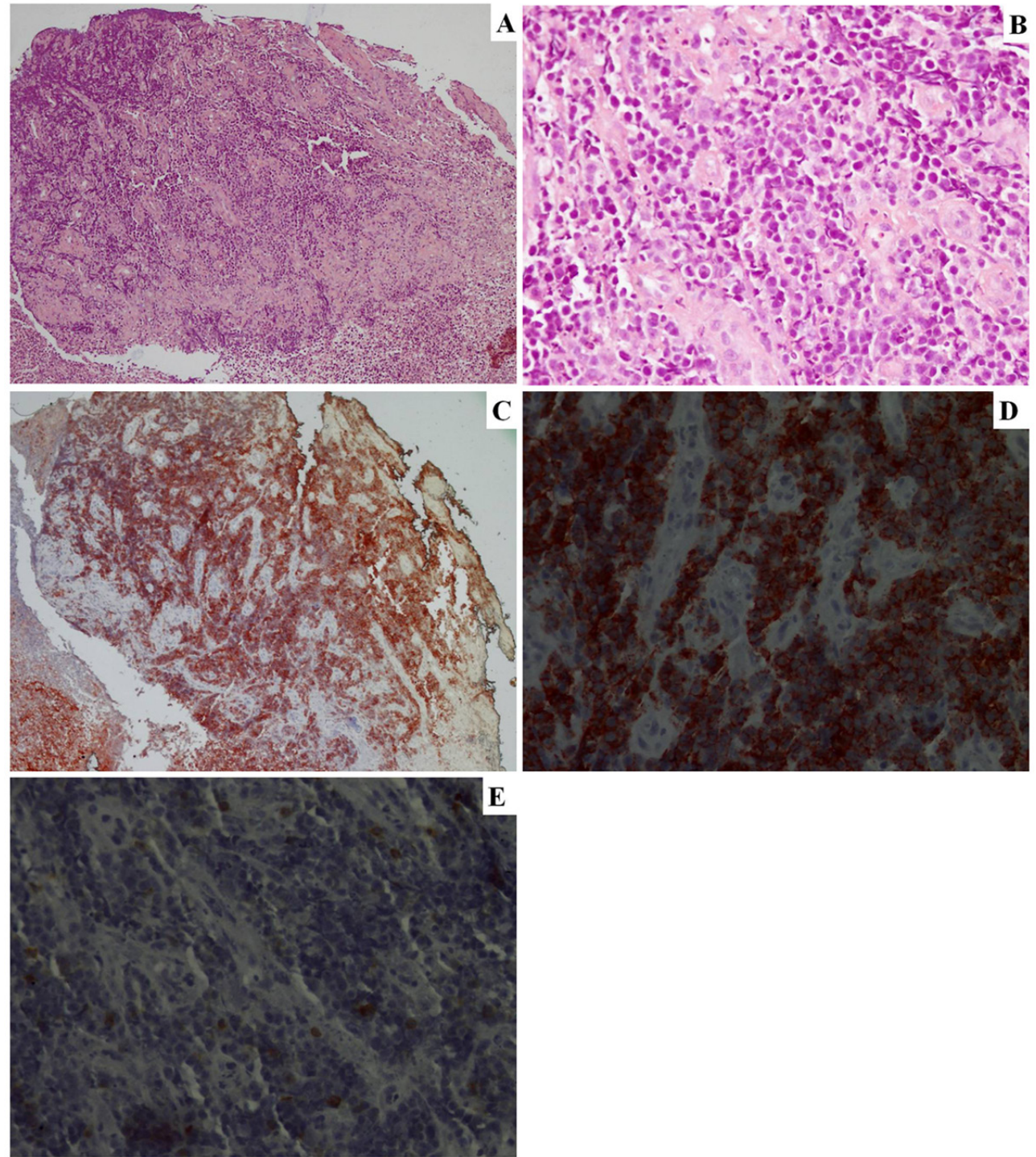

Figure 3. Histological examination of endoscopic biopsy specimens showing atypical lymphoid cell infiltration at submucosa and lamina propria. (A) Hematoxilyn-eosin staining (× 100); (B) Hematoxilyn-eosin staining ( $\times 400)$; (C) CD20(+) (× 100); (D) CD20(+) (× 400); (E) CD3(-) (× 400). 
also multiple lymphadenopathies adjacent to the mass lesion. Colonoscopic examination was consistent with an obstructive, ulcerated and necrotic mass lesion at the neighborhood of cecum (Fig. 2). Biopsies obtained during the colonoscopy were consistent with diffuse large B-cell lymphoma (Fig. 3). The patient underwent right hemicolectomy with primary anastamosis, and then, was treated with systemic chemotherapy.

\section{Discussion}

Intussusception is the telescopic invagination of intestinal segment into the adjacent distal part of small intestine or large intestine. Adult intussusception cause $1-5 \%$ of all adult intestinal obstructions [4]. Most of adult intussusception cases (approximately 90\%) are secondary to other benign or malignant disorders with a lead point lesion. The etiologic factor might be a benign lesion like, fibroid polyp, lipoma, Meckel's diverticulum, Crohn's disease and Yersinia enterocolitis or a malignant tumor such as a primary carcinoma, metastatic carcinoma such as melanoma, GISTs, lymphoma, or carcinoid tumor $[3,5,6]$. In contrast to pediatric intussusception cases most of which are primary and benign, most of adult patients have structural lesions, especially malignancy in colonic cases [7]. Patients present with nonspecific symptoms such as nausea, vomiting, gastrointestinal bleeding, change in bowel habits, constipation or abdominal distension [8]. Although majority of adult cases have a chronic intermittent course, some patients present with acute or subacute symptoms. Classical clinical triad can be detected in about $10-20 \%$ of adult cases $[7,9]$. On the other hand, although CT can detect a lead point easily on $\mathrm{CT}$ examination, determination of intussusception cause is not always possible with CT imaging, except lipoma [10]. Vague presentation of intussusception in adulthood causes delaying of the diagnosis.

Primary colonic lymphomas account for only $0.2-0.6 \%$ of colon cancers and $10-20 \%$ of all gastrointestinal lymphomas [11]. Colonic lymphomas are found more frequently in males in their sixth and seventh decade of life. Diffuse large B-cell lymphoma and mucosa-associated lymphoid tissue lymphoma are the most common histological subtypes. The most frequently involved colonic site at diagnosis is cecum followed by the sigmoid and the rectum. Clinical presentation of colonic lymphoma is quite vague and variable. Intussusception is very rarely seen in intestinal lymphoma. The most common type of lymphoma causing intussusception is diffuse B-cell lymphoma [12].

In our patient, diffuse B-cell lymphoma causing ileocolonic intussusception is the case. The patient attended to our clinic with nonspecific abdominal pain and intermittent bloody diarrhea. There was no fever, night sweat or weight loss and laboratory finding was not remarkable except LDH. Moreover, amebiasis complicated the case and led to delay in diagnosis. The lymphoma was not obviously demonstrated on CT imaging. The patient was diagnosed accurately with colonoscopic examination and pathological findings after the treatment of $E$. histolytica infection with metronidazole.

Ileocolonic or colocolonic intussusception is extremely rare presentation of intestinal amebiasis [13]. Some infections cause intestinal intussusception. It is suggested that infection-related mesenteric lymph node involvement or mass formation may constitute lead point for intussusception development. On the other hand, intestinal amebiasis may present with bloody diarrhea, abdominal pain or intestinal mass lesion (ameboma). In the presence of stool E. histolytica antigen positivity, bloody diarrhea and abdominal pain symptoms are suggestive for invasive amebic colitis. Persistence of abdominal pain after resolving of bloody diarrhea gave an impression about other presumptive diagnosis.

Consequently, intussusception has a course with indistinct symptoms. It is mandatory to define the cause, especially in adult patients. Intestinal amebiasis may cause similar symptoms with intussusception. However, clinical course of intestinal amebiasis resembles intussusception symptoms and this tropical infection may masquerade colonic lymphoma.

\section{Conflict of Interest}

The authors declare that there is no conflict of interests regarding the publication of this paper.

\section{Grant Support}

None.

\section{References}

1. Haas EM, Etter EL, Ellis S, Taylor TV. Adult intussusception. Am J Surg. 2003;186(1):75-76.

2. Stubenbord WT, Thorbjarnarson B. Intussusception in adults. Ann Surg. 1970;172(2):306-310.

3. Akbulut S. Unusual cause of adult intussusception: diffuse large B-cell non-Hodgkin's lymphoma: a case report and review. Eur Rev Med Pharmacol Sci. 2012;16(14):1938-1946.

4. Azar T, Berger DL. Adult intussusception. Ann Surg. 1997;226(2):134-138.

5. Rabbani K, Narjis Y, Jgounni R, Semlani Z, Difaa A, Benelkhaiat R, Louzi A, et al. Adult intussusception caused by an inflammatory fibroid ileal polyp. Acta Chir Belg. 2012;112(2):157-159.

6. Cantarella S, Zisa M, Grasso E, Politi A, Guastella T. The intussusception in patients with Crohn's disease: the 
role of the surgeon. Updates Surg. 2013;65(1):77-80.

7. Yalamarthi S, Smith RC. Adult intussusception: case reports and review of literature. Postgrad Med J. 2005;81(953):174-177.

8. Marinis A, Yiallourou A, Samanides L, Dafnios N, Anastasopoulos G, Vassiliou I, Theodosopoulos T. Intussusception of the bowel in adults: a review. World J Gastroenterol. 2009;15(4):407-411.

9. Wang N, Cui XY, Liu Y, Long J, Xu YH, Guo RX, Guo KJ. Adult intussusception: a retrospective review of 41 cases. World J Gastroenterol. 2009;15(26):3303-3308.

10. Kim YH, Blake MA, Harisinghani MG, Archer-Arroyo K, Hahn PF, Pitman MB, Mueller PR. Adult intestinal intussusception: CT appearances and identification of a causative lead point. Radiographics. 2006;26(3):733-744.

11. Konstantinidis IT, Probstfeld MR. Lymphoma presenting as a necrotic colonic mass. World J Gastrointest Surg. 2012;4(4):102-103.

12. Li B, Shi YK, He XH, Zou SM, Zhou SY, Dong M, Yang JL, et al. Primary non-Hodgkin lymphomas in the small and large intestine: clinicopathological characteristics and management of 40 patients. Int J Hematol. 2008;87(4):375-381.

13. Patra SB, Giri DD, Shukla GN, Mehta HS. Amoebic granuloma--an unusual cause of caeco-colic intussusception. Postgrad Med J. 1984;60(700):168-170. 